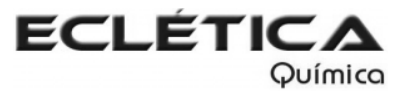

www.scielo.br/eq

Volume 31, número 1, 2006

\title{
Determinação de parâmetros de validação de métodos cromatográficos para análise de 5-hidroximetilfurfural e açúcares em amostras de processo de produção de polímero biodegradável
}

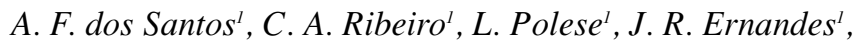 \\ S. M. Kesserlingh ${ }^{2}$, R. V. Nonato ${ }^{2}$ \\ ${ }^{\prime}$ Instituto de Química - UNESP - 14801-970 - Araraquara - SP \\ ${ }^{2}$ PHB Industrial S/A - 14150-000 - Serrana - SP
}

\begin{abstract}
Resumo: A eficiência de métodos para análise de 5-hidroximetilfurfural por cromatografia líquida de alta eficiência com detecção na região do ultravioleta e determinação de sacarose, glicose e frutose por cromatografia líquida com detecção por índice de refração foi avaliada. Após otimização das condições analíticas, os principais parâmetros de validação (linearidade, limite de quantificação, limite de detecção, recuperação, sensibilidade e precisão) foram determinados e demonstraram que os procedimentos analíticos podem ser aplicados para o controle do processo de produção de poli(3-hidroxibutirato).
\end{abstract}

Palavras-chave: cromatografia líquida; açúcares; 5-hidroximetilfurfural; poli(3-hidroxibutirato).

\section{Introdução}

Poli(hidroxialcanoatos) (PHAs) são polímeros termoplásticos biodegradáveis produzidos mediante processos biotecnológicos a partir de fontes de carboidratos renováveis e constituem uma classe geral de polímeros microbianos [1], que em bactérias são produzidos e acumulados intracelularmente, na forma de grânulos, como reserva de carbono e energia, podendo apresentar até $80 \%$ da massa seca total da célula [2].

O poli(3-hidroxibutirato) (PHB) é um dos PHAs mais estudados e possui propriedades termoplásticas semelhantes às do polipropileno. Entre os microrganismos que produzem PHB, a espécie Ralstonia eutropha é uma das que apresentam as condições mais favoráveis para produção em escala industrial [2].
A produção industrial de $\mathrm{PHB}$ tem sido realizada empregando como substrato açúcar invertido [1]. A inversão da sacarose é uma etapa extremamente importante na produção de PHB, pois a linhagem da bactéria (Ralstonia eutropha) empregada não assimila diretamente a sacarose, mas sim glicose e frutose [3]. Além disso, no processo de inversão pode ser produzido o 5hidroximetilfurfural (5-HMF) que é tóxico para a Ralstonia eutropha. Portanto, torna-se imprescindível o desenvolvimento e/ou a validação de métodos analíticos para avaliar a eficiência da inversão da sacarose e a concentração de 5-HMF no substrato alimentar das bactérias.

Este trabalho descreve a otimização das condições experimentais e a determinação dos principais parâmetros de validação de métodos empregando cromatografia líquida de alta efi- 
ciência (HPLC) com detecção na região do ultravioleta (UV) para análise de 5-HMF e HPLC com detecção por índice de refração (RI) para a determinação simultânea de sacarose, glicose e frutose.

\section{Materiais e métodos}

\section{Equipamentos}

HPLC - RI

Cromatógrafo líquido equipado com duas bombas modelo ProStar 210 (Varian), injetor manual modelo 7125 (Rheodyne) com loop de $20 \mu \mathrm{l}$, detector de índice de refração modelo R401 (Waters) e integrador modelo Chromatopac C-R6A (Shimadzu). Coluna analítica de aço inox KS-801 (300 mm x 8 mm d.i.; Shodex) e pré-coluna de aço inox KS-G (Shodex) foram empregadas para determinação de sacarose, glicose e frutose. Condições de operação: temperatura da coluna, $22 \pm 2^{\circ} \mathrm{C}$; polaridade, positiva; atenuação, 8; velocidade do papel, 2,5 mm/min; fase móvel, solução de hidróxido de sódio $\left(5,0\right.$. 10 10 $\left.\mathrm{mol} \mathrm{L}^{-1}\right)$ com vazão de 0,4 $\mathrm{ml} / \mathrm{min}$.

\section{HPLC - UV}

Cromatógrafo líquido equipado com duas bombas modelo ProStar 210 (Varian), injetor manual modelo 7125 (Rheodyne) com loop de $20 \mu \mathrm{l}$ e detector UV-Vis modelo 320 (Varian) foi usado para análise de 5-HMF. Coluna analítica de aço inox C18 (250 mm x $4.6 \mathrm{~mm}$ d.i.., $5 \mu \mathrm{m}$; Varian) foi mantida a $22 \pm 2^{\circ} \mathrm{C}$. A fase móvel consistiu de acetonitrila:água $(5: 95, \mathrm{v} / \mathrm{v})$ a $1 \mathrm{ml} / \mathrm{min}$. A detecção foi realizada a $283 \mathrm{~nm}$. A aquisição e o tratamento de dados foram realizados com Star Chromatography Workstation (Varian).

\section{Reagentes, solventes e materiais}

Acetonitrila (J.T.Baker) foi de grau HPLC. Sacarose (Labsynth), glicose (Reagen), frutose (Sigma) e hidróxido de sódio (Mallinckrodt Baker) foram de grau analítico. Padrão de 5-HMF (99\%) foi obtido da SigmaAldrich. Soluções estoque foram preparadas a partir dos padrões de carboidratos ou 5-HMF em água para obtenção de concentração de $7 \mathrm{~g} \mathrm{~kg}^{-1}$ (sacarose), $15 \mathrm{~g} \mathrm{~kg}^{-1}$ (glicose), $24 \mathrm{~g} \mathrm{~kg}^{-1}$ (frutose) e 300 $\mathrm{mg} \mathrm{kg}^{-1}$ (5-HMF). As soluções de trabalho foram preparadas mediante diluição das soluções estoque. Solução de hidróxido de sódio $\left(5,0 \cdot 10^{-4} \mathrm{~mol} \mathrm{~L}^{-1}\right)$ e acetonitrila:água (5:95, v/v) usadas como fase móvel, amostras, soluções de carboidratos e 5-HMF foram preparadas com água purificada em sistema Milli-Q (Millipore) e filtradas em membranas com porosidade de 0,45 $\mu \mathrm{m}$ (Millipore).

\section{Métodos}

Determinação de açúcares por HPLC-RI

Para determinação de açúcares em amostra líquida (xarope) construiu-se uma curva de resposta do sinal analítico (área) contra concentração dos padrões de sacarose, glicose e frutose para verificar a linearidade do detector no intervalo de concentração de trabalho, porém a quantificação pode ser feita por fator de resposta.

Massas de padrão de sacarose, glicose e frutose foram pesadas e diluídas em água relação massa/massa $(\mathrm{m} / \mathrm{m})$ - e após filtração foram analisadas por HPLC-RI.

As respectivas concentrações de sacarose, glicose e frutose podem ser determinadas diluindo-se as amostras de xarope em água respeitando-se o intervalo de linearidade do detector e $o$ limite de quantificação.

\section{Determinação de 5-HMF por HPLC-UV}

Para determinação de 5-hidroximetilfurfural (5-HMF) em amostra líquida (xarope) construiu-se uma curva de resposta do sinal analítico (área) contra concentração do padrão de 5-HMF para verificar a linearidade do detector no intervalo de concentração de trabalho e a quantificação faz-se com fator de resposta.

Alíquotas de padrão de 5-HMF foram pesadas e diluídas em água - relação massa/massa $(\mathrm{m} / \mathrm{m})$ - e após filtração foram analisadas por HPLC-UV. A detecção foi realizada no comprimento de onda de absorção máxima do 5-HMF, ou seja, 283nm.

A concentração de 5-HMF pode ser determinada diluindo-se as amostras de xarope em água considerando-se o intervalo de linearidade do detector e o limite de quantificação do método. 


\section{Resultados e discussão}

As condições experimentais tais como: composição e vazão da fase móvel foram otimizadas considerando o tempo de análise e a separação adequada dos compostos de interesse. Inicialmente, metanol:água foi testado como fase móvel para determinação de 5-HMF. Entretanto, o uso de acetonitrila:água forneceu uma maior simetria do pico cromatográfico e sob as condições experimentais descritas o tempo de análise foi de aproximadamente 15 minutos. Métodos têm sido descritos para determinação de açúcares empregando gradiente de eluição e detector amperométrico. Entretanto, o detector de índice de refração requer o uso de análise isocrática, além do controle de temperatura. A vazão da fase móvel para análise de açúcares foi selecionada considerando o limite de pressão (<715 psi) recomendado pelo fabricante da coluna utilizada e mantendo-se as condições mencionadas a separação de sacarose, glicose e frutose foi realizada em aproximadamente 30 minutos. Cromatogramas das análises de sacarose, glicose e frutose por HPLC-RI e 5-HMF por HPLC-UV estão apresentados na Figura 1.

Com o intuito de garantir a confiabilidade dos resultados analíticos foram determinados os principais parâmetros de validação dos procedimentos propostos para a análise de 5-HMF e açúcares. Devido a biocompatibilidade do PHB, a determinação dos parâmetros de validação (sensibilidade, intervalo de trabalho, linearidade, precisão, recuperação, limite de detecção e limite de quantificação) das metodologias descritas para o
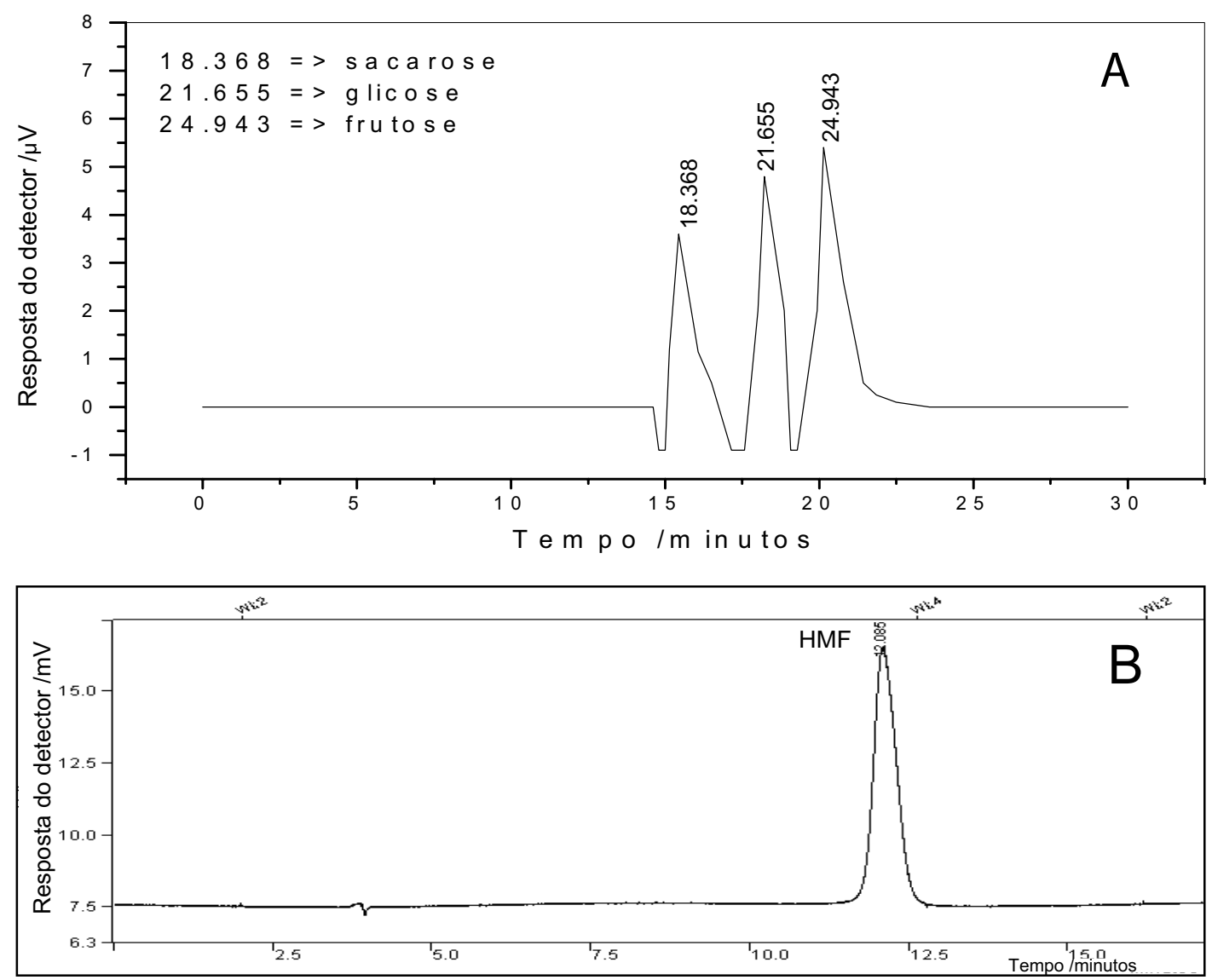

Figura 1. Cromatogramas: (A) solução padrão de sacarose, glicose e frutose; (B) solução padrão de 5HMF. 
controle do processo de produção do PHB foi realizada de acordo com as recomendações da Agência Nacional de Vigilância Sanitária ANVISA [4] para métodos cromatográficos em amostras de fármacos e outros produtos com aplicações farmacêuticas.
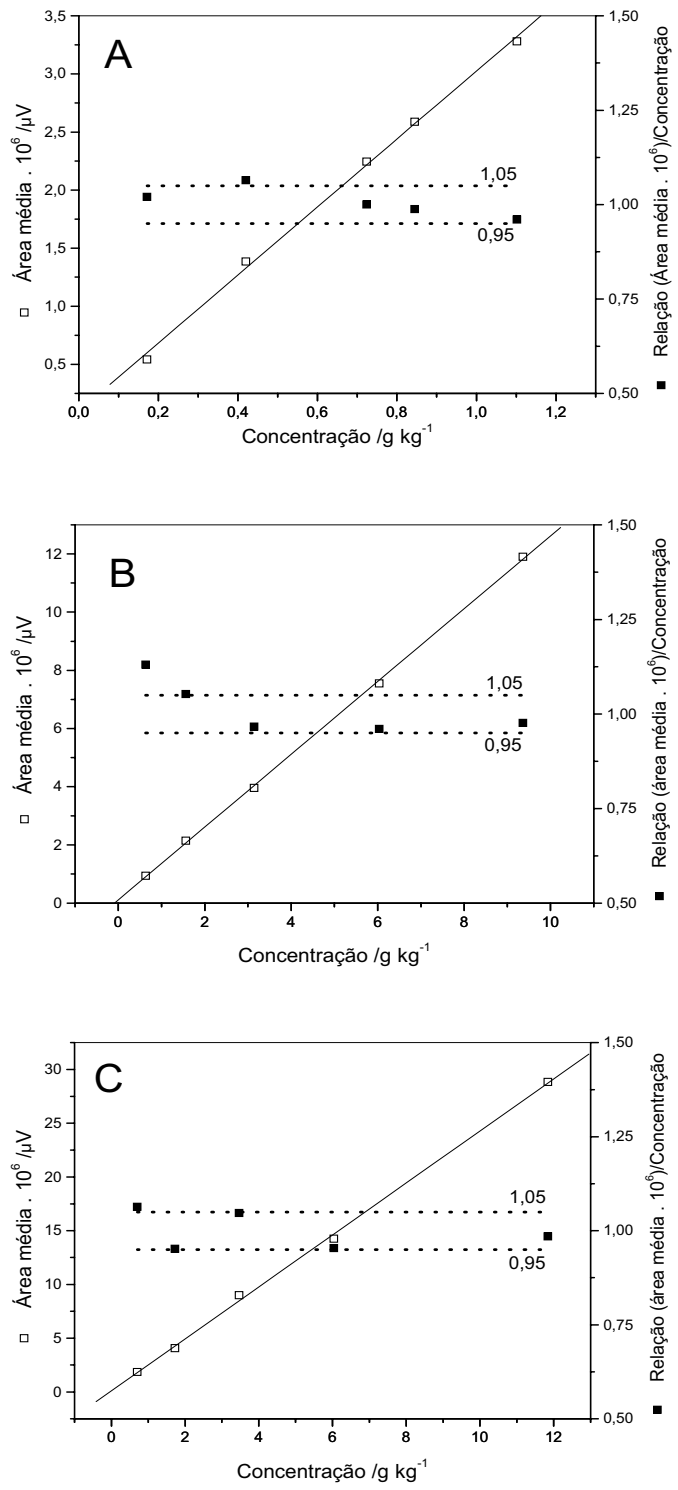

Figura 2. Área média e relação de área média sobre concentração em função da concentração das soluções de padrão: (A) sacarose; (B) glicose; (C) frutose.
A análise dos açúcares (sacarose, glicose e frutose) foi realizada simultaneamente empregando fator de resposta para cada substância, porém para verificar a linearidade do detector, no intervalo de concentração esperado nas amostras do processo de inversão da sacarose, foram injetadas no HPLC-RI em triplicata cinco soluções de diferentes concentrações dos padrões dos carboidratos. Com injeções de soluções contendo individualmente cada um dos analitos de interesse deste método, fez-se a identificação por meio dos específicos tempos de retenção dos picos cromatográficos. A média dos correspondentes tempos de retenção para sacarose, glicose e frutose com os valores de desvio padrão (DP, n=3) foram 18,27 $\pm 0,02,21,65 \pm 0,05$ e $24,7 \pm 0,5$ minutos. Valores de coeficiente de variação $(\mathrm{CV}, \mathrm{n}=3)$ inferiores a 7,6 $\%$ foram obtidos para os valores de área dos picos cromatográficos referentes aos carboidratos. Estes resultados demonstram a precisão instrumental.

A Figura 2 que apresenta a distribuição dos valores de área média e relação de área média sobre concentração em função da concentração dos correspondentes açúcares.

Os fatores de resposta $\left(F R_{n}\right)$ calculados segundo a Equação 1 e seus valores de desvio padrão $(n=3)$ para sacarose, glicose e frutose estão dispostos na Tabela 1.

$$
F R_{n}=\frac{A_{m}}{c}
$$

Na equação $1 A_{m}$ corresponde aos valores de área dos açúcares, $c$ a concentração e $F R_{n}$ o fator de resposta com índice $n$ subscrito denotando os açúcares.

Os valores de sensibilidade (dado pelo coeficiente angular da reta), limite de quantificação, limite de detecção, intervalo de trabalho, precisão, recuperação média e os respectivos valores de desvio padrão $(n=3)$ obtidos para análises realizadas em três níveis de concentração estão dispostos na Tabela 1.

Os valores obtidos de relação da área média sobre concentração (respostas relativas) em função da concentração dos correspondentes açúcares (FIGURA 2) demonstram a linearidade no intervalo de concentração de trabalho, pois com exceção do menor valor de concentração de glicose, as respostas relativas apresentam varia- 
Tabela 1. Parâmetros das curvas de resposta para determinação da concentração de sacarose, glicose e frutose.

\begin{tabular}{|c|c|c|c|}
\hline \multirow{2}{*}{ PARÂMETRO } & & & \multirow[b]{2}{*}{ FRU } \\
\hline & SAC & $\begin{array}{r}\text { GLI } \\
\text { VALORES } \\
\end{array}$ & \\
\hline Sensibilidade $/ \mu \mathrm{V} / \mathrm{g} \mathrm{kg}^{-1}$ & $2,9295 \cdot 10^{6}$ & $1,2511 \cdot 10^{6}$ & $2,4175 \cdot 10^{6}$ \\
\hline Intervalo de trabalho $/ \mathrm{g} \mathrm{kg}^{-1}$ & $0,1712-1,1017$ & $0,6371-9,3592$ & $0,7045-11,8453$ \\
\hline $\begin{array}{l}\text { Precisão } \\
\text { (CV,repetitividade/\%) }\end{array}$ & 4,71 & 3,92 & 3,60 \\
\hline \multirow{3}{*}{$\begin{array}{l}\text { Recuperação (\%) } \\
\text { Média } \pm \text { DP }\end{array}$} & $0,2635 \mathrm{~g} \mathrm{~kg}^{-1}=>94 \pm 5$ & $1,3530 \mathrm{~g} \mathrm{~kg}^{-1}=>98 \pm 3$ & $1,7256 \mathrm{~g} \mathrm{~kg}^{-1} \Rightarrow>101 \pm 3$ \\
\hline & $0,6573 \mathrm{~g} \mathrm{~kg}^{-1}=>95 \pm 5$ & $2,4338 \mathrm{~g} \mathrm{~kg}^{-1}=>103 \pm 6$ & $5,3731 \mathrm{~g} \mathrm{~kg}^{-1} \Rightarrow>102 \pm 4$ \\
\hline & $0,9768 \mathrm{~g} \mathrm{~kg}^{-1}=>96 \pm 8$ & $3,1440 \mathrm{~g} \mathrm{~kg}^{-1}=>98 \pm 3$ & $14,8980 \mathrm{~g} \mathrm{~kg}^{-1} \Rightarrow>101 \pm 5$ \\
\hline $\mathrm{LQ} / \mathrm{g} \mathrm{kg}^{-1}$ & 0,0512 & 0,1223 & 0,0728 \\
\hline $\mathrm{LD} / \mathrm{g} \mathrm{kg}^{-1}$ & 0,0154 & 0,0367 & 0,0218 \\
\hline FR $\cdot 10^{6} \pm \mathrm{DP}$ & $3,1 \pm 0,1$ & $1,3 \pm 0,1$ & $2,5 \pm 0,1$ \\
\hline
\end{tabular}

ção de $\pm 5 \%$ [5]. Proporcionalmente tem-se uma maior sensibilidade para a sacarose, pois para uma pequena variação de concentração de sacarose ocorre uma maior variação de área em relação aos outros açúcares.Os valores de precisão (repetitividade) da relação de área dos picos cromatográficos no nível médio de concentração, dados pelo coeficiente de variação $(n=3)$ para sacarose, glicose e frutose foram 4,71; 3,92 e $3,60 \%$, respectivamente. Valores médios $(n=3)$ de recuperação entre 94 e $103 \%$ com desvio padrão entre 3 e 8 foram obtidos para os açúcares nos três níveis de concentração avaliados. Estes resultados estão de acordo com os limites $(5,6<$ $\mathrm{CV}<8$ ) aceitos para o intervalo de concentração estudado [6]. Os valores de limite de quantificação (LQ) determinados a partir da Equação 2 para sacarose, glicose e frutose foram 0,0512 , 0,1223 e $0,0728 \mathrm{~g} \mathrm{~kg}^{-1}$, respectivamente.

$L Q=\frac{D P_{c} 10}{I C}$

$\mathrm{Na}$ Equação 2, $\mathrm{DP}_{\mathrm{c}}$ representa o desvio padrão para no mínimo três curvas de resposta e IC o valor do coeficiente angular da reta, considerando uma curva de resposta linear.

Os valores de limite de detecção (LD) forma determinados a partir da Equação 3.
$D=\frac{D P_{c} 3}{I C}$

Portanto, na determinação simultânea da concentração de sacarose, glicose e frutose em amostras do processo (xarope) pode-se utilizar uma equação equivalente a Equação 1, já que são obtidos os valores do fator de resposta dos padrões e os valores de área dos picos cromatográficos dos respectivos açúcares presentes nas amostras.

A linearidade da resposta do detector para o 5-HMF foi determinada, no intervalo de con-

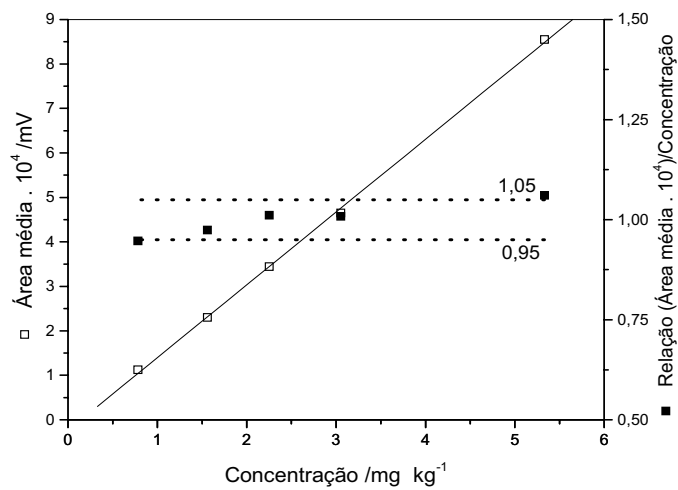

Figura 3. Área média e relação de área média sobre concentração de 5-HMF em função das diferentes concentrações das soluções de padrão de 5-HMF. 
Tabela 2. Parâmetros da curva de resposta de 5-HMF.

\begin{tabular}{lr}
\hline PARÂMETRO & \multicolumn{1}{c}{ VALORES } \\
\hline Sensibilidade $/ \mathrm{mV} / \mathrm{mg} \mathrm{kg}^{-1}$ & $1,5627.10^{11}$ \\
Intervalo de trabalho $/ \mathrm{mg} \mathrm{kg}^{-1}$ & $0,7839-7,3326$ \\
Precisão & 1,07 \\
(CV,repetitividade/\%) & $1,3884 \mathrm{mg} \mathrm{kg}^{-1}=>101 \pm 1$ \\
Recuperação (\%) & $2,0573 \mathrm{mg} \mathrm{kg}^{-1}=>102 \pm 1$ \\
média $\pm \mathrm{DP}$ & $2,7648 \mathrm{mg} \mathrm{kg}^{-1}=>101 \pm 3$ \\
$\mathrm{LQ} / \mathrm{mg} \mathrm{kg}^{-1}$ & 0,2098 \\
$\mathrm{LD} / \mathrm{mg} \mathrm{kg}^{-1}$ & 0,0629 \\
$\mathrm{FR} \cdot 10^{11} \pm \mathrm{DP}$ & $1,51 \pm 0,07$ \\
\hline
\end{tabular}

centração de trabalho para as amostras, mediante injeções dos cinco padrões em triplicata no mesmo dia. Valores de coeficiente de variação $(\mathrm{n}=3)$ inferiores a $4,5 \%$ foram obtidos para a área do pico cromatográfico. A média dos tempos de retenção e o desvio padrão $(\mathrm{n}=3)$ calculado foi $12,18 \pm 0,04$ minuto. Estes resultados mostram a precisão instrumental.

A distribuição dos pontos para a média de área e a relação de área média sobre concentração em função da concentração de 5-HMF estão apresentadas na Figura 3. Observa-se proporcionalidade da resposta do detector no intervalo de concentração estudado.

$\mathrm{O}$ resultado médio do fator de resposta $\left(F R_{n}\right)$ e o respectivo desvio padrão $(\mathrm{n}=3)$ para 5HMF estão apresentados na Tabela 2. O valor de $F R_{n}$ foi calculado para soluções com concentrações de $0,7839 \mathrm{mg} \mathrm{kg}^{-1}$ a $5,3326 \mathrm{mg} \mathrm{kg}^{-1}$.

A partir dos valores de $F R_{n}$ dos padrões e área (resposta do detector) obtidos para 5-HMF em amostras de xarope, resultantes da inversão da sacarose determina-se a concentração do analíto de interesse empregando-se a Equação 1.

Os valores de limite de quantificação, limite de deteç̧ão, sensibilidade, intervalo de trabalho, precisão, recuperação média e os valores de desvio padrão obtidos para análises realizadas em três níveis de concentração estão dispostos na Tabela 2.

O limite de quantificação (LQ) determinado foi de $0,2098 \mathrm{mg} \mathrm{kg}^{-1}$ e o limite de detecção foi de $0,0629 \mathrm{mg} \mathrm{kg}^{-1}$. Os valores médios $(\mathrm{n}=3)$ de recuperação variaram de 101 a $102 \%$ com desvio padrão entre 1 e 3 nos três niveis de concentração estudados e estão de acordo com os valores recomendados, ou seja CV menor que 16 [6].

Embora a eficiência de uma metodologia analítica deva ser continuamente avaliada, os parâmetros de validação determinados permitem que os métodos descritos sejam aplicados em amostras para o controle do processo de produção de PHB.

\section{Conclusão}

A otimização das condições experimentais e a determinação dos principais parâmetros de validação demonstraram que os métodos cromatográficos propostos podem ser empregados para a análise de 5-hidroximetifurfural e açúcares (sacarose, glicose e frutose) em amostras do processo de produção do poli(3-hidroxibutirato).

\section{Agradecimentos}

Ao convênio estabelecido entre a Fundação de Apoio à Ciência, Tecnologia e Educação (FACTE) e a PHB Industrial S/A.

Recebido em: 14/09/2005

Aceito em : 08/12/2005 
A. F. dos Santos, C. A. Ribeiro, L. Polese, J. R. Ernandes, S. M. Kesserlingh, R. V. Nonato. Determination of validation parameters to chromatographic methods in 5-hydroxymethilfurfurol and sugar analysis to samples from production process of biodegradable polymer.

Abstract: The efficiency of methods for the analysis of 5-hydroxymethylfurfural by high performance liquid chromatography with ultraviolet detection (HPLC-UV) and determination of sucrose, glucose and fructose by HPLC with refractive index detection (HPLC-RI) was evaluated. After optimization of the analytical conditions, the main validation parameters (linearity, quantification limit, detection limit, recovery, sensitivity and precision) were determined and demonstrated that the analytical procedures could be applied to control the process of poly(3-hydroxybutyrate) production.

Keywords: liquid chromatography; sugars; 5-hydroxymethylfurfural; poly(3-hydroxybutyrate)

\section{Referências}

[1] J. G. C. Gomez, C.L. Bueno Netto, Rev. Bras. Eng. Quím., 17(1997)24

[2] E.A. Dawes, P.J. Senior. Advances In Microbial Physiology. Academic Press California, 10(1973)136.

[3] C.R. Squio, G.M.F. Aragão. Quím. Nova, 27(2004)615.
[4] Agencia Nacional de Vigilância Sanitária. Resol. n.899, de 23 de maio de 2003. D. O. U., 02 jun. 2003. in: $<$ http://www.anvisa.gov.br/legis/resol/2003/re/899 03re.htm $>$. [5] M. Ribani, C.B.G. Bottoli, C.H. Collins, I.C.S.F. Jardim, L.F.C. Melo, Quím. Nova, 27(2004)771.

[6] R. Wood, Trends Anal. Chem., 18(1999)624. 